\title{
Endostatin reverses immunosuppression of the tumor microenvironment in lung carcinoma
}

\author{
XIAOLIN LIU $^{1 *}$, WEIWEI NIE $^{1 *}$, QI XIE $^{2}$, GUOLING CHEN $^{3}$, XINGYU LI $^{1}$, \\ YANRUI JIA ${ }^{1}$, BEIBEI YIN ${ }^{1}, \mathrm{XUN} \mathrm{QU}^{4}$, YAN LI $^{1}$ and JING LIANG ${ }^{1}$ \\ ${ }^{1}$ Department of Oncology; ${ }^{2}$ Medical Research Center, Qianfoshan Hospital, Shandong University, \\ Jinan, Shandong 250014, P.R. China; ${ }^{3}$ Islet Cell Laboratory, MedStar Georgetown University Hospital, Washington, \\ DC 20007, USA; ${ }^{4}$ Institute of Basic Medical Sciences and Key Laboratory of Cardiovascular Proteomics \\ of Shandong Qilu Hospital, Shandong University, Jinan, Shandong 250012, P.R. China
}

Received September 2, 2016; Accepted May 31, 2017

DOI: $10.3892 / \mathrm{ol} .2017 .7455$

\begin{abstract}
Endostatin has previously been demonstrated to efficiently inhibit the angiogenesis and growth of endothelial cells. However, the role of endostatin in the tumor microenvironment remains to be elucidated. To investigate the antitumor effect of endostatin in lung cancer, the present study was designed to explore the alterations of microvessel density in Lewis lung cancer models and the expression of vascular endothelial growth factor (VEGF), interleukin (IL)-6, IL-17, interferon (IFN) $-\gamma$ and hypoxia inducible factor (HIF)- $1 \alpha$, following endostatin therapy. It was demonstrated that the growth and angiogenesis of tumors were markedly suppressed by treatment with endostatin, compared with control group. The microvessel density in mice treated with endostatin was significantly inhibited in a dose-dependent manner. The expression levels of VEGF, IL-6 and IL-17 in tumors were decreased, however IFN- $\gamma$ and HIF-1 $\alpha$ expression levels were increased, following treatment with endostatin. In addition, the proportion of myeloid derived suppressor cells and tumor associated macrophages (TAMs; M2 type) were significantly decreased, whereas those of mature dendritic cells and TAMs (M1 type) were increased, and cluster of differentiation (CD) $8^{+}$ $\mathrm{T}$ cells were recruited to infiltrate the tumors following treatment with endostatin. In addition, the expression levels of IL-6, IL-10, tumor growth factor- $\beta$ and IL-17 in tumor tissue were potently decreased with endostatin therapy. These results indicated that endostatin efficiently inhibited tumor angiogenesis
\end{abstract}

Correspondence to: Professor Jing Liang, Department of Oncology, Qianfoshan Hospital, Shandong University, 16766 Jingshi Road, Jinan, Shandong 250014, P.R. China

E-mail: liangjing0531@163.com

*Contributed equally

Key words: endostatin, lung cancer, cellular therapy, tumor microenvironment and reversed the immunosuppressive microenvironment associated with the presence of tumors.

\section{Introduction}

Inflammation plays crucial roles in the development of tumor. Tumor is regarded as a never healed injury (1). It has been proven that immune inflammatory cell could enhance angiogenesis, accelerate cell proliferation, invasion and metastasis in tumor development (2). Tumor can recruit normal cells to build tumor microenvironment. Inflammatory reaction stimulates angiogenesis and tissue reconstitution to promote the progress of cancer (3).

Angiogenesis is one of the crucial hallmarks of tumor. Recent studies have indicated that multiple mechanisms were involved in anti-angiogenesis therapy, such as immunity (4-6). It is important to optimize tumor treatment strategies according to the estimation of the change of tumor microenvironment after anti-angiogenesis therapy. Several studies powerfully supported that anti-angiogenesis therapy can overcome various suppressive immunity network (7). Sunitinib can reduce the number of MDSCs, Tregs and the expressions of IL-10, TGF- $\beta$ and PD-1 to relieve the immunity suppression in tumor (8). In the nude mouse models, neutralizing VEGFR can arrest VEGF signaling pathway, promote DCs maturation and downregulate the number of Tregs. In view of stablization of endothelial cells, targeting angiogenesis is one prospective method to control process of tumor. Endostatin is considered with be no side effects and less multidrug resistance, which could efficiently inhibit the angiogenesis and the growth of endothelial cells (9). However, there is rare research of endostatin in the tumor microenvironment.

In our previous study, we had proven that endostatin plays antitumor effect combined with DC-T cell therapy in lung carcinoma. To investigate further mechanism of endostatin antitumor effect, we explored the effect of endostatin on immune cells and cytokines in tumor microenvironment, and the intervention of the immune network, aiming to offer potential data for molecular targeted therapy or adoptive cellular immunotherapy. 


\section{Materials and methods}

Cells. Lewis lung cancer cell line (from lung adenocarcinoma cell line of C57BL/6 mice) was purchased from Shanghai Cell Bank of Chinese Academy of Sciences and was cultured in Dulbecco's modified Eagle's medium (DMEM) containing $10 \%$ fetal bovine serum. The cells $\left(1 \times 10^{7}\right)$ were resuspended in RPMI-1640 medium (Thermo Fisher Scientific, Waltham, MA, USA) and mixed.

Animals. Male wild-type C57BL/6 mice (age, 6 weeks; weight, 18-22 g) were purchased from Beijing Laboratory Animal Center of Chinese Academy of Sciences and fed in the specific-pathogen-free animal laboratory. The feeding and use of laboratory animals complied with Animal Experimentation Ethical Standards proposed by Ethics Committee of Shandong University [SCXK (Lu) 2003-0003].

After Lewis lung carcinoma cells (LLCs) were recovered and subcultured in complete medium, cells in log growth phase were used and cell concentration was adjusted to $1 \times 10^{7} / \mathrm{ml}$. Right rib skin of C57BL/6 mice was disinfected with $75 \%$ alcohol and suspension of LLCs was collected with $1 \mathrm{ml}$ syringe (mixing upside down). Suspension $(0.2 \mathrm{ml})$ was then given to each mouse via subcutaneous injection, with $1 \times 10^{6}$ cells being inoculated in each mouse.

C57BL/6 mice were divided into three groups (low dose of endostatin, high dose of endostatin and PBS control) with 7 mice in each group. Intervention was given to tumor-bearing mice on day 7 . Tumor growth and diameter were measured every other day. The mice were killed after $24 \mathrm{~h}$ with administration on day 14 . For control group, phosphate-buffered saline $(0.2 \mathrm{ml})$ was given to each C57BL/6 mouse by tail vein injection for a total of 14 days; for low dose of endostatin group, was given to each mouse by tail vein injection for a total of 14 days at a concentration of $7.5 \mathrm{mg} / \mathrm{kg} /$ day; for high dose of endostatin were given $15 \mathrm{mg} / \mathrm{kg} /$ day to each mouse by tail vein injection on day 7 after the model of tumor-bearing C57BL/6 mice was established.

To measure mouse weight and tumor inhibition rate, electronic scale was used to measure the weight of mice in each group every other day. After tumor-bearing C57BL/6 mice were killed, tumor tissues were dissected and weighed. Tumor inhibition rate $=(1-$ mean tumor weight of treatment groups/mean tumor weight of control group) x $100 \%$. For the measurement of tumor volume, the maximum long and short diameters of tumors were measured using vernier caliper. Then, mean tumor volume and tumor inhibition rate in each group were calculated and growth curve was drawn. Tumor volume $=$ long diameter of tumor $\mathrm{x}$ short diameter of tumor ${ }^{2} / 2$.

Antibodies and reagents. Recombinant human endostatin (rhEndostatin; Simcere Pharm, Nanjing, China); EZ-Sep ${ }^{\mathrm{TM}}$ mouse percollase (Amresco, Dallas, TX, USA); RPMI-1640 medium, FBS (Gibco, Grand Island, NY, USA); ConA, DMEM medium, phosphate-buffered saline (PBS) buffer (Sigma, St. Louis, MO, USA); fluorescently-labeled antibody CD3, CD4, CD8, CD11c, CD86, major histocompatibility complex (MHC) II, CD11b, Gr-1, CD206, CD68 and NOS2 and their isotype controls (eBioscience, Inc., San Diego, CA, USA); mouse lymphocyte factor ELISA kit (Shanghai
Enzyme-linked Biotechnology Co., Ltd., Shanghai, China); BCA Protein Assay kit (Beyotime, Shanghai, China); anti-mouse hypoxia-inducible factor- $1 \alpha$ (HIF-1 $\alpha)$, VEGF antibody (Abcam, Cambridge, MA, USA); rmGM-CSF, rmIL-4 (Peprotech), rmIL-2, rmTNF- $\alpha$ (Biological); anti-mouse CD31 nonlabeled immunohistochemical monoclonal antibody (Santa Cruz Biotechnology, Inc., Santa Cruz, CA, USA); MCO-15AC $\mathrm{CO}_{2}$ incubator (Sanyo, Osaka, Japan); sterile 1.5 laminar flow bechtop (Thermo Fisher Scientific); FACSCalibur flow cytometer (Becton-Dickinson, Franklin Lakes, NJ, USA); NanoDrop ND-1000 ultraviolet spectrophotometer (Agilent Technologies, Inc., Santa Clara, CA, USA); Model 680 Microplate Reader (Bio-Rad, Hercules, CA, USA).

Immunohistochemistry. Tumor tissues were separated from tumor-bearing mice and fixed with paraformaldehyde. The tissues were embedded with paraffin and then sectioned. The paraffin sections were dewaxed to water. The antigens were repaired by high pressure for 8 min using streptavidin-peroxidase method. Subsequent procedures were conducted according to instructions of secondary antibody kit and DAB color development kit (Bio-Rad). Under a light microscope, mean microvessel density (MVD) was counted under 6 high power fields (x200) for each section.

Flow cytometry. Tumor tissues were dissected and cut into pieces. After trypsinization, the tissues were filtered through $300 \mathrm{~mm}$ stainless steel mesh to obtain monocytes. After cell density was adjusted to $5 \times 10^{5} / \mathrm{ml}, 100 \mu \mathrm{l}$ cell suspension was added into each flow tube. FACS tubes contained phycoerythrin-labeled anti-CD83 and anti-CD86 antibodies (BioLegend, San Diego, CA, USA), allophycocyanin-labeled anti-CD68 antibody, phycoerythrin-labeled anti-iNOS antiboody, fluorescein isothiocyanate-labeled anti-CD3, anti-CD4, anti-IFN- $\gamma$, anti-CD206, anti-Gr-1 and anti-CD11c antibodies (eBioscience, Inc.). Negative control, isotype control and single line pipette groups were designed. Phycoerythrin-rat IgG and fluorescein isothiocyanate-hamster IgG were added in control groups. After being fully mixed with fluorescent-labeled antibody, they were placed at room temperature away from light for 15 min. FACS Aria II sorting flow cytometer (BD Biosciences, San Jose, CA, USA) was used to detect molecule expressions on cell surface and FlowJo software was used to analyze data.

Enzyme linked immunosorbent assay (ELISA). Antibodies were diluted with coating buffer to the extent where protein level was $1-10 \mu \mathrm{g} / \mathrm{ml}$. Then, $0.1 \mathrm{ml}$ of antibodies were taken and added to ELISA plate wells, which were incubated at $4^{\circ} \mathrm{C}$ overnight. The required number of wells was calculated according to the number of test samples, blank control and standard samples. Distilled water $(130 \mu \mathrm{l})$ was added into blank and standard wells, while $100 \mu \mathrm{l}$ cell supernatant was added into sample wells for testing. Each sample group was made in triplicate. The plate was covered and incubated in an incubator with $5 \% \mathrm{CO}_{2}$ at $37^{\circ} \mathrm{C}$ for $90 \mathrm{~min}$. Afterwards, liquid in wells was discarded and the wells were washed 3 times. After $100 \mu \mathrm{l}$ biotin antibody was added to each well, the plate was covered and incubated in an incubator with $5 \% \mathrm{CO}_{2}$ at $37^{\circ} \mathrm{C}$ for $1 \mathrm{~h}$. Then, the plate was washed with washing liquid 
for 3 times of $3 \mathrm{~min}$. Enzyme conjugate $(100 \mu \mathrm{l})$ was added into each well and the plate was incubated in the incubator with 5\% $\mathrm{CO}_{2}$ at $37^{\circ} \mathrm{C}$ for $30 \mathrm{~min}$. Afterwards, the plate was washed for 3 times. Color developing reagent $(100 \mu \mathrm{l})$ was added into each well and kept away from light. The plate was incubated in the incubator with $5 \% \mathrm{CO}_{2}$ at $37^{\circ} \mathrm{C}$ for $10-20 \mathrm{~min}$. Finally, $100 \mu \mathrm{l}$ stop buffer was added into each well to terminate reaction and OD450 values were determined using a microplate reader (Model 680; Bio-Rad, Hercules, CA, USA).

Western blotting. After tumor tissues were taken out from tumor-bearing mice. They were chipped and then ground in a homogenizer. Total protein was extracted through disruption on ice, and $400 \mu 1$ RIPA lysate was added to each group. After disruption, cells were taken out and transferred to a centrifugal tube of $1.5 \mathrm{ml}$. Then, cells were centrifuged at $13,200 \mathrm{x} \mathrm{g}$ at $4^{\circ} \mathrm{C}$ for $20 \mathrm{~min}$, and the supernatant was collected. Bicinchoninic Acid Protein Assay kit was used to determine the concentration of extracted protein (Beyotime). Sodium dodecyl sulfate polyacrylamide gel electrophoresis was used to transfer protein to polyvinylidene fluoride film. The film was then covered with Tris-buffered saline and Tween-20 (TBST) containing 5\% skimmed milk for $2 \mathrm{~h}$. After primary antibodies (rabbit anti-mouse HIF-1 $\alpha$ monoclonal antibody, 1:1,000; rabbit anti-mouse VEGF monoclonal antibody, 1:1,000; Abcam) were added, the film was incubated at $4^{\circ} \mathrm{C}$ overnight. The film was washed five times (6 min each) with TBST. After secondary antibody (horseradish peroxidase goat anti-mouse IgG antibody conjugate, 1:2,000; Abcam) was added, the film was incubated at $37^{\circ} \mathrm{C}$ for $1 \mathrm{~h}$ and washed with TBST. Electrochemiluminescence kit was used to achieve chemiluminescence signals. SmartView electrophoresis image analysis system (Smartview Enterprise Imaging Solutions, Irvine, CA, USA) was used to obtain images. Quantity One software (Bio-Rad) was applied to analyze gray values of each zone. The ratio of each interested protein and gray values of $\beta$-actin was calculated for statistical analysis.

Immunohistochemisty. Tumor tissues were separated from tumor-bearing mice and fixed with formalin. The tissues were embedded with paraffin and then sectioned. The paraffin sections were dewaxed to water. Streptavidin-peroxidase method was used following kit instructions. Primary antibodies were replaced with phosphate-buffered saline as negative control. According to semi-quantitative integration, the images were reviewed by two physicians from the Department of Pathology and a conclusion was drawn. The results were scored according to positive staining intensity and expression of positive cells. Negative staining intensity: cells had no staining (score 0 ); light brown cells were weakly positive (score 1); brown cells were moderately positive (score 2); brown cells without background coloring were strongly positive (score 3). For expression of positive cells, 5 different fields were chosen under $x 400$ light microscope and 200 cells were counted for each field. The percentage of positive cells was then calculated. If positive cells $\leq 5 \%$, score was 0 ; if positive cells $\leq 25 \%$, score was 1 ; if $25 \%<$ positive cells $\leq 50 \%$, score was 2 ; if positive cells $>50 \%$, score was 3 . There were four grades of immunohistochemisty results according the above scores: 0 was rated as negative (-), 1-4 were rated as weakly positive (+), 5-8 were graded as moderately positive (+), and 9-12 were rated as strongly positive (+++).

Statistical analysis. All data were analyzed using SPSS 18.0 software (IBM, New York, NY, USA). Measurement data were expressed as means \pm SD. Statistical differences between groups were determined using one-way ANOVA, and LSD test was performed to make comparison between any two groups. Ration comparison was conducted by $\chi^{2}$ test and Fisher's exact test. $\mathrm{P}<0.05$ was considered to indicate a statistically significant difference.

\section{Results}

Tumor growth and angiogenesis were strongly inhibited by endostatin therapy. To test the effect of endostatin therapy on tumor growth and angiogenesis, tumor sizes were measured and immunohistochemistry (IHC) were performed separately. Compared with control group, tumor growth was actively suppressed by endostatin therapy $(\mathrm{P}=0.013)$, while there was no difference of tumor growth between low and high dose group of endostatin ( $\mathrm{P}>0.05)$ (Fig. $1 \mathrm{~A}$ and $\mathrm{B})$. Using $\mathrm{CD} 31$ as the marker of vascular endothelial cells, cytoplasm of endothelial cells was stained with yellowish-brown. In contract to control group, tumor MVD in mice receiving low dose of endostatin was fewer $(\mathrm{P}=0.014)$, while MVD in mice receiving tumor high dose of endostatin was significantly decreased $(\mathrm{P}=0.002)$, and the necrosis of tumor tissues were also increased, which was different between these two groups $(\mathrm{P}<0.05)$ (Fig. 1C and D). These results indicate by dose dependent, endostatin markedly inhibited tumor angiogenesis, promoted tumor necrosis and significantly reduced tumor growth.

Factors of tumor angiogenesis were strongly inhibited by endostatin therapy. To test the effect of endostatin therapy on tumor angiogenesis and hypoxia, IHC were performed separately. Compared with control group, the expression of VEGF was actively suppressed by low dose of endostatin therapy $(\mathrm{P}=0.001)$, while the expression of HIF-1 $\alpha$ was significantly increased $(\mathrm{P}=0.000)$. Similarly, the expression of VEGF was lower and the expression of HIF-1 $\alpha$ was increased markedly in high dose of endostatin therapy ( $\mathrm{P}=0.001)$ (Fig. 2A-C). Expressions of IL- 6 and IL-17 were reduced $(\mathrm{P}=0.012$; $\mathrm{P}=0.029)$, and the expression of IFN- $\gamma$ was increased strongly $(\mathrm{P}=0.044)$ in low dose of endostatin therapy by detection with ELISA. Similarly, the expressions of IL-6 and IL-17 was decreased strongly $(\mathrm{P}=0.012 ; \mathrm{P}=0.029)$ and the expression of IFN- $\gamma$ was increased strongly $(\mathrm{P}=0.036)$ in high dose of endostatin therapy (Fig. 2D). These data proved that endostatin exacerbated hypoxic conditions and inhibited proangiogenic factors and increased expressions of antiangiogenic factors.

Endostatin effectively decreased the infiltration of immunosuppression cell and promoted infiltration of DCs and CD8+T cells in tumor. Compared to the controls, the proportion of MDSCs was reduced in low dose $(\mathrm{P}=0.035)$ and less in high dose of endostatin $(\mathrm{P}=0.019)$. Immune-enhanced M1 type of TAMs were increased in low dose $(\mathrm{P}=0.031)$ and more in high dose $(\mathrm{P}=0.017)$. Immunity suppressive $\mathrm{M} 2$ type of TAMs were 
A

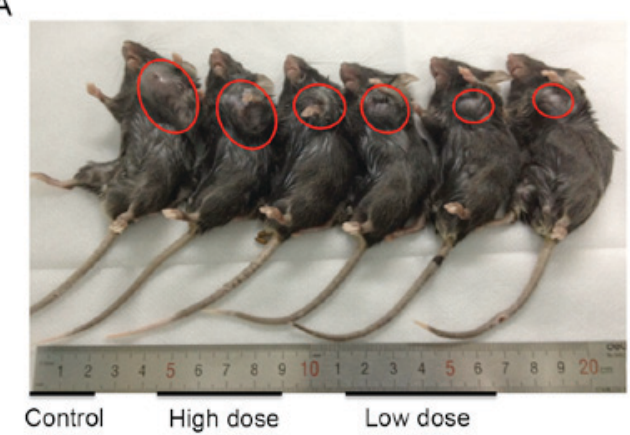

B

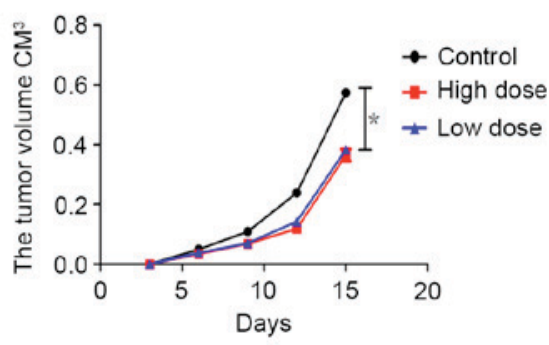

$\mathrm{C}$

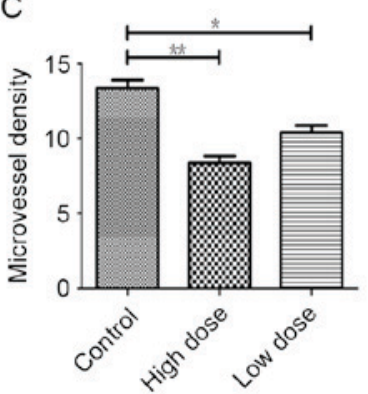

$D$

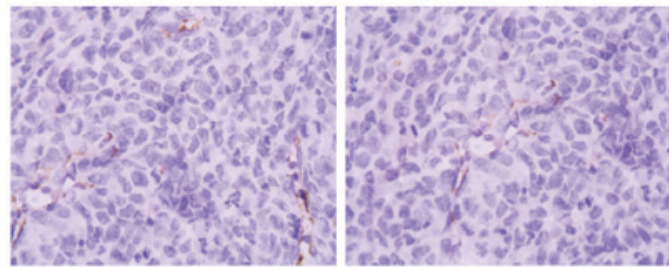

Control

High dose

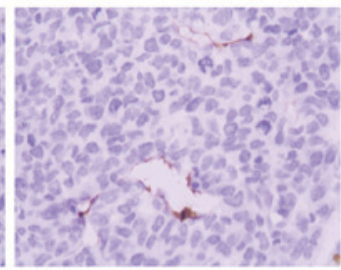

Low dose

Figure 1. Antitumor and anti-vascular effect of Endostatin on established LLC tumors. (A and B) The tumors were dissected and the tumor growth curves are shown. (C and D) The microvessel density (MVD) is determined by blinded measurement of CD31 expression. The data shown are representative results of independent experiments in the figure, $\mathrm{n}=7$ per group for each experiment. ${ }^{*} \mathrm{P}<0.05 ;{ }^{* *} \mathrm{P}<0.01$.

A

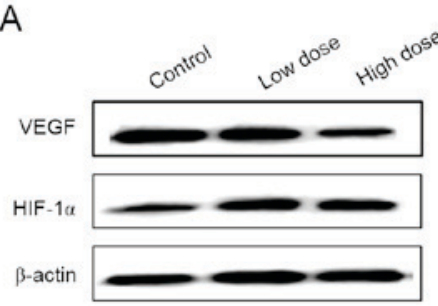

${ }^{B}$
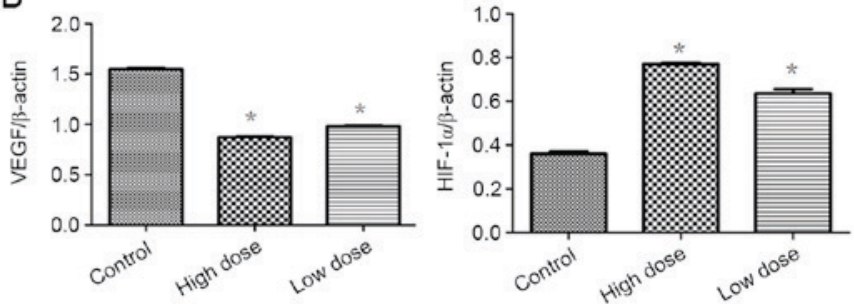

C
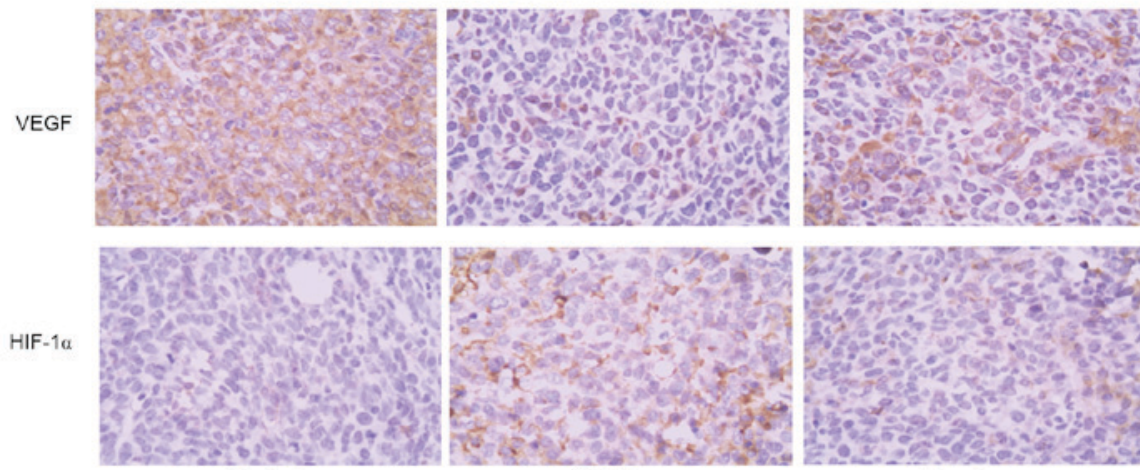

Control

D
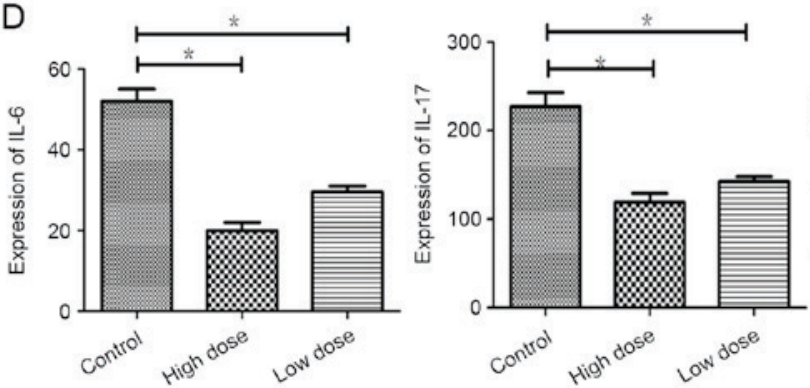

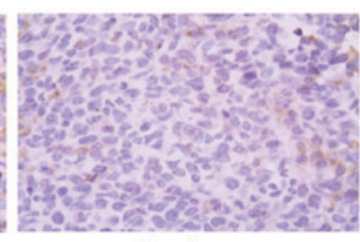

Low dose

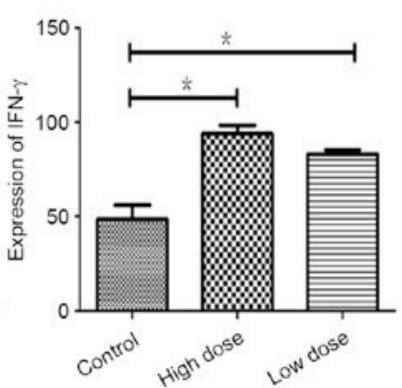

Figure 2. Endostatin efficiently descreased multiple proangiogenic factors, and augmented hypoxia in the tumors. (A and B) The VEGF and HIF-1 $\alpha$ expressions in the tumors were showed. The expression of $\beta$-actin served as an internal control. (C) The expression of VEGF and HIF-1 $\alpha$ in the tumors. (D) Expression of the pro/anti-antigiogenic cytokines IL-6, IL-17 and IFN- $\gamma$. Data are expressed as the mean \pm SD of three independent experiments. * $<<0.05$. 

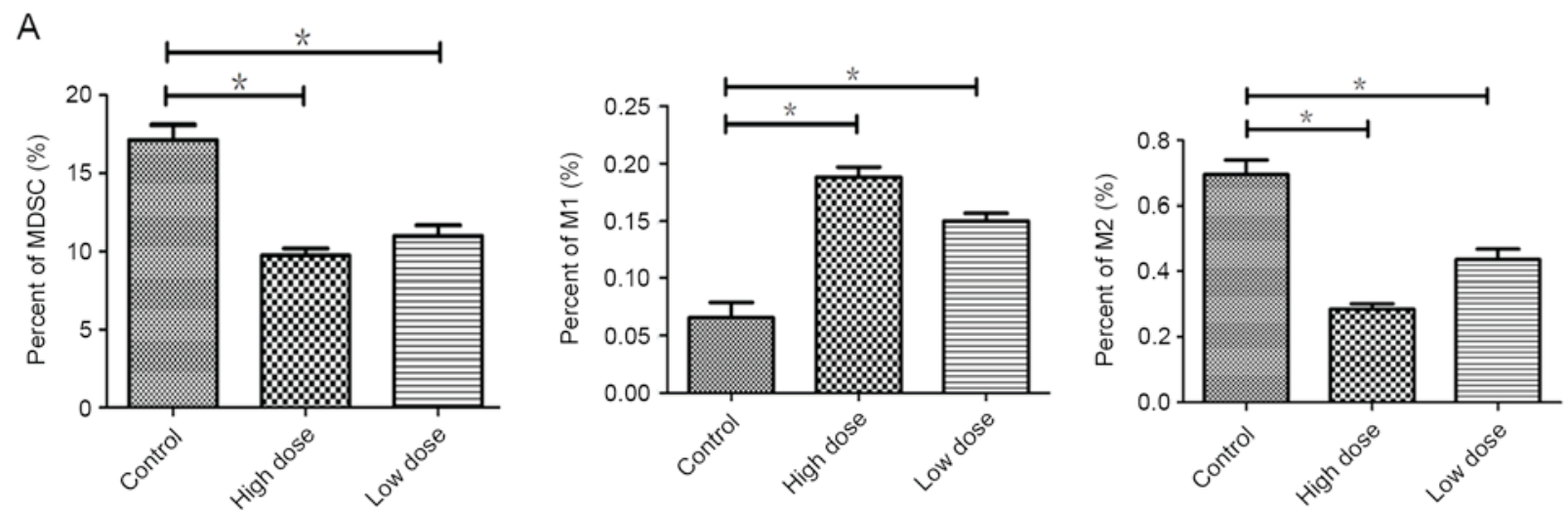

B
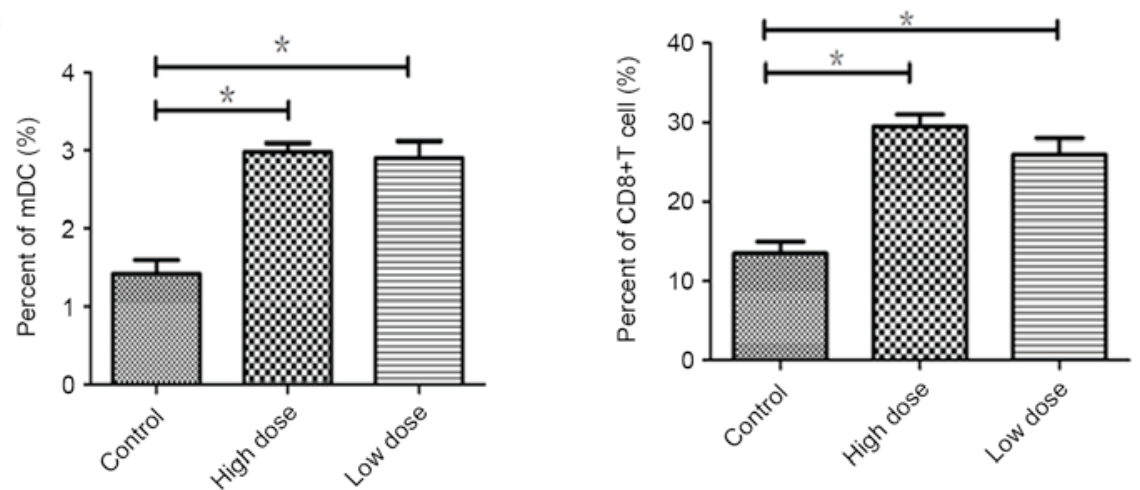

Figure 3. Endostatin discreased inhibitory immune cells in the tumor microenvironment and increased tumor-infiltrated mDCs and CD8 ${ }^{+} \mathrm{T}$ cells. (A) The immunosuppressive MDSC and TAM are significantly lower. (B) The mDCs are upregulated, and cytotoxic CD8 ${ }^{+} \mathrm{T}$ cells are recruited to infiltrate the tumors by endostatin. Data expressed as the mean \pm SD are representative of three independent experiments, using tumor pooled from seven animals per group. ${ }^{*} \mathrm{P}<0.05$.

declined in low dose $(\mathrm{P}=0.042)$ and more in high dose group $(\mathrm{P}=0.01)$ (Fig. 3A).

Compare to the control, the infiltration of $\mathrm{mDCs}$ and $\mathrm{CD}^{+} \mathrm{T}$ cells were increased in low dose $(\mathrm{P}=0.034 ; \mathrm{P}=0.038)$, which were significantly increased in the high dose group $(\mathrm{P}=0.018 ; \mathrm{P}=0.017)$ (Fig. 3B). There was no significant difference between low and high dose group $(\mathrm{P}>0.05)$. The study suggested that through downregulation of immunosuppressive cells, endostatin enchanced M1 type of TAMs, the cell infiltration of mature DCs and $\mathrm{CD} 8^{+} \mathrm{T}$ cells in tumor, to reverse immunosuppression of tumor micro-environment.

Endostatin increased the expression of IFN- $\gamma$ by inhibition of immunosuppressive cytokines in tumor. Compare to the control, immunosuppressive cytokines including IL-6, IL-10, IL-17 and TGF- $\beta$ were reduced significantly $(\mathrm{P}=0.022,0.020,0.038,0.018)$ in low dose of endostatin by detected with ELISA, which was more significant in high dose group ( $\mathrm{P}=0.012,0.018,0.014,0.029)$. The expression of IFN- $\gamma$ was increased $(\mathrm{P}=0.044)$, which was more in high dose of endostatin $(\mathrm{P}=0.036)$. There was no significant difference between low and high dose ( $\mathrm{P}>0.05)$ (Fig. 4). The study suggested that endostatin repressed the expressions of immunosuppressive factors to inhibit immunosuppression of tumor micro-environment.

Our study proved that endostatin could inhibit the expressions of immunosuppressive cells and cytokines, enhance the M1 type of TAMs and IFN- $\gamma$, and accelerate the invasion of mature DCs and $\mathrm{CD}^{+} \mathrm{T}$ cells in tumor, reversing immunosuppression of tumor micro-environment, and play antitumor effect collaboration with DC-T cell therapy.

\section{Discussion}

Studies have proved that inflammation play crucial roles in the development of cancer. Inflammatory cells can prove angiogenesis, accelerate cell proliferation, invasion and migration (10). As one critical factor of tumor, angiogenesis promotes tumor growth and migration to surrounding tissue, and even spread to other organ (11). Folkman suggested that it is a good way to inhibit tumor growth through arrest development of new vascular formation (12). Endothelial cell can be regarded as target of antitumor therapy. Angiogenesis is produced by interaction of various type of cells in tumor microenviroment (13). Inflammation can promote tumor development by angiogenesis and organization restructuring (2).

Inflammatory microenvironment is major structure of all the tumors (14). About $90 \%$ of tumor are related with mutation of somatic cell and environment factor. In other words, cancer can be a never healed wound. TAMs and T cells are most common in tumor microenvironment. TAMs could enhance tumor growth, angiogenesis, invasion and migration. Angiogenesis is dependent on recruitment of TAMs to angiopoietin 2 and VEGF (15). CXCL8/IL-8, CXCL1, VEGF and HIF- $1 \alpha$ could be regulated by TAMs, MDSCs, NF- $\kappa$ B, STAT3 and AP-1 $(4,16)$. The inactivation of NF- $\kappa b$ and STAT3, neutralization of CCL2 or CXCL12, section of TAMs, inhibit angiogenesis, arrest tumor growth, which 

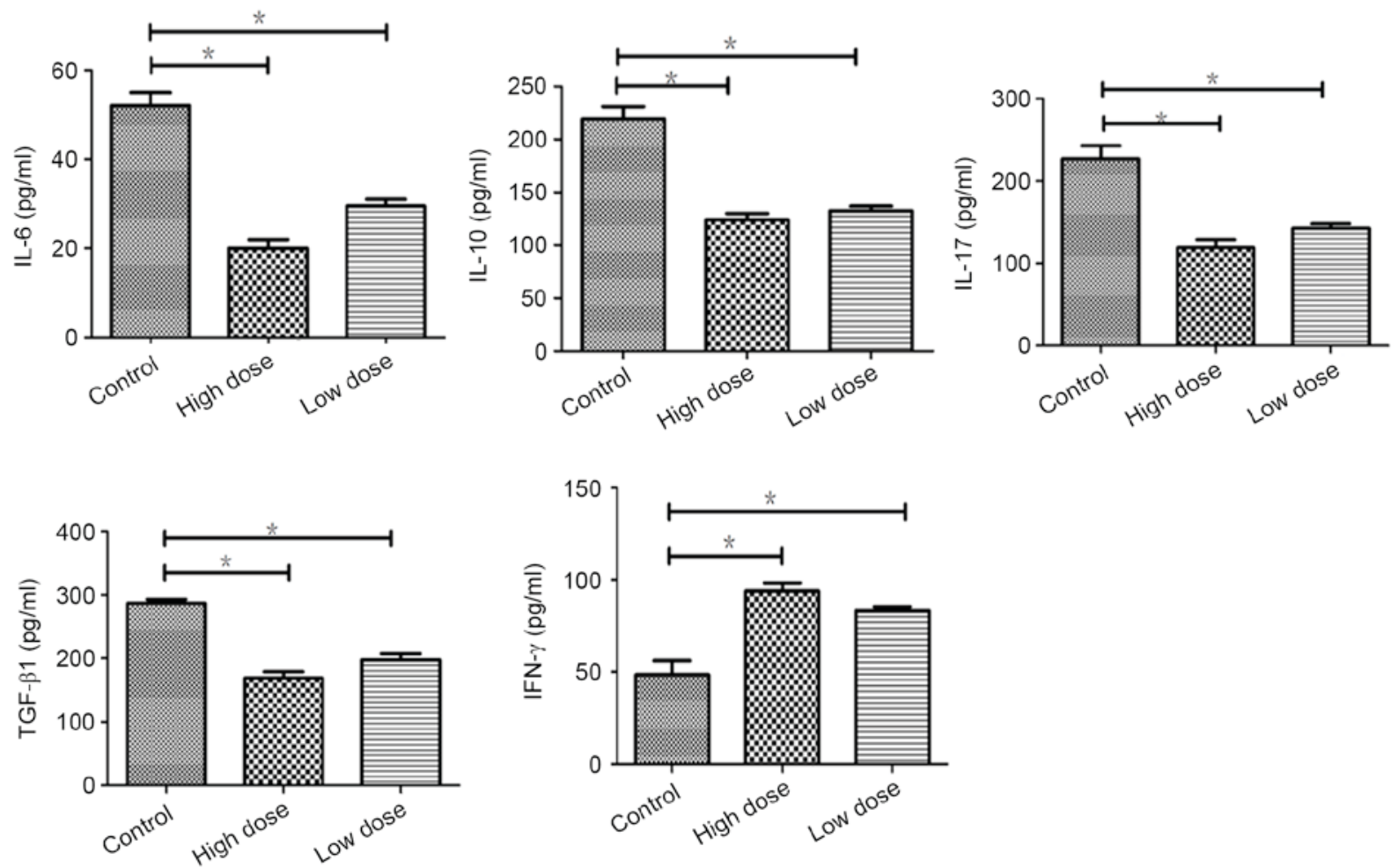

Figure 4. Endostatin decreased immune inhibitory cytokines in the tumor microvironment. Endostatin significantly reduced the expression of IL6, IL-10, TGF- $\beta$ and IL-17 in the tumor tissue, and concurrently elevated IFN- $\gamma$ in comparison with control group. Data expressed as the mean \pm SD are representative of three independent experiments, using tumor pooled from seven animals per group. " $\mathrm{P}<0.05$.

indicate the critical role of inflammation medium in tumor angiogenesis (17). There are all kinds of partial differentiation of myeloid progenitor cells in tumor stroma, which have the preference to promote tumor formation (18). Myeloid-derived suppressor cells (MDSC) are a major host component contributing to the immune suppressive environment. In addition to their inherent immune suppressive function, MDSC amplify the immune suppressive activity of macrophages and dendritic cells via cross-talk $(2,19,20)$. Hypoxia, a reduction in the normal level of tissue oxygen tension, occurs during acute and chronic vascular disease, pulmonary disease and cancer (21). The chemokine CXCL12 and its receptor CXCR4 are known to play an important role in cancer development and progression (22). HIF-1 $\alpha$ could enhance the expression of CXCL12, which activates endothelial cells apoptosis a induced by overexpression CXCR4 (23). HIF-1 $\alpha$ also induced the expression of VEGF in hypoxic condition and angiogenesis by collecting bone marrow inhibitory cells from different sources $(24,25)$.

Given that the gene stability of endothelial cells, it has been a good way to inhibit the progress of tumor by targeting angiogenesis (11). As one of endogenous inhibitor, endostatin was found in epithelioid hemangioendothelioma (26). By its regulation to angiogenesis gene, endostatin can inhibit proliferation, migration or invasion and apoptosis of endothelial cells, to play antineoplastic activity (27). It has been approved to cure or retreatment III/IV stage non-small cell lung cancer combined with NP chemotherapy (NP regimen includes Navelbine $\left(25 \mathrm{mg} / \mathrm{m}^{2}, \mathrm{~d} 1, \mathrm{~d} 8\right)$ and DDP $\left(25 \mathrm{mg} / \mathrm{m}^{2}, \mathrm{~d} 1-3\right)$, repeated every 21 days) in September of 2005 by China's state food and drug administration. Studies have suggested that VEGF inhibit the maturation of DCs by regulation of VEGFR-1 induced $\kappa \mathrm{B}$ dependent signal $(28,29)$. mDCs from peripheral blood of cancer patients are associated with increased serum levels of VEGF. Therefore, antiangiogenic therapy blocking VEGF pathway, promote DCs maturation and the decline of Tregs numbers.

In our study, we found that endostatin reduced the fraction of immunosuppressive MDSCs and M2 type of TAMs in Lewis lung mouse model. The expressions of immunosuppressive factors including IL-6, IL-10, IL-1 and TGF- $\beta$ were downregulated by endostatin. M1 type of TAMs and IFN- $\gamma$ were upregulated by endostatin, which promote invasion of mature DCs and $\mathrm{CD}^{+} \mathrm{T}$ cell in tumor microenvironment. These results proved endostatin effectively corrects immunosuppression of tumor microenvironment. The mechanism about the effect of endostatin to tumor microenvironment is rare. To explore the specific mechanism of endostatin antitumor effect, and its intervention to tumor microenvironment immune network, we can carry out therapy combined endostatin and cellular immunotherapy to find theoretical basis. On the other hand, our study have found that endostatin inhibited IL-6, IL-17 and VEGF, upregulated the expressions of IFN- $\gamma$, which supports that endostatin inhibits angiogenesis and enhances hypoxia in tumor microenvironment. Our previous study have proved that endostatin enhances the antitumor effect of DC-T cell immunity, and corrects the immunity inhibition, which suggested endostatin has close relationship with body immune system. Therefore, to study the mechanism of endostatin antitumor, we will offer further therapy data to find predictive markers with target therapy and cell immunity. 


\section{Acknowledgements}

This study was supported by the Natural Science Youth Foundation of Shandong Province, P.R. China (no. ZR2013HQ017) and the Shandong Provincial Natural Science Foundation, China (no. ZR2010HL015; ZR2015HL024).

\section{Reference}

1. Hanahan D and Weinberg RA: Hallmarks of cancer: The next generation. Cell 144: 646-674, 2011.

2. Prueitt RL, Wallace TA, Glynn SA, Yi M, Tang W, Luo J, Dorsey TH, Stagliano KE, Gillespie JW, Hudson RS, et al: An immune-inflammation gene expression signature in prostate tumors of smokers. Cancer Res 76: 1055-1065, 2016.

3. Yuan Y, Jiang YC, Sun CK and Chen QM: Role of the tumor microenvironment in tumor progression and the clinical applications (Review). Oncol Rep 35: 2499-2515, 2016.

4. Ham M and Moon A: Inflammatory and microenvironmental factors involved in breast cancer progression. Arch Pharm Res 36: 1419-1431, 2013.

5. Samples J, Willis M and Klauber-Demore N: Targeting angiogenesis and the tumor microenvironment. Surg Oncol Clin N Am 22: 629-639, 2013.

6. Jayson GC, Kerbel R, Ellis LM and Harris AL: Antiangiogenic therapy in oncology: Current status and future directions. Lancet 388: 518-529, 2016.

7. Tartour E, Pere H, Maillere B, Terme M, Merillon N, Taieb J, Sandoval F, Quintin-Colonna F, Lacerda K, Karadimou A, et al: Angiogenesis and immunity: A bidirectional link potentially relevant for the monitoring of antiangiogenic therapy and the development of novel therapeutic combination with immunotherapy. Cancer Metastasis Rev 30: 83-95, 2011.

8. Chen HM, Ma G, Gildener-Leapman N, Eisenstein S, Coakley BA, Ozao J, Mandeli J, Divino C, Schwartz M, Sung M, et al: Myeloid-derived suppressor cells as an immune parameter in patients with concurrent sunitinib and stereotactic body radiotherapy. Clin Cancer Res 21: 4073-4085, 2015.

9. Walia A, Yang JF, Huang YH, Rosenblatt MI, Chang JH and Azar DT: Endostatin's emerging roles in angiogenesis, lymphangiogenesis, disease, and clinical applications. Biochim Biophys Acta 1850: 2422-2438, 2015.

10. Green DR, Galluzzi L and Kroemer G: Mitochondria and the autophagy-inflammation-cell death axis in organismal aging. Science 333: 1109-1112, 2011.

11. Potente M, Gerhardt H and Carmeliet P: Basic and therapeutic aspects of angiogenesis. Cell 146: 873-887, 2011

12. Ackermann M and Konerding MA: Vascular casting for the study of vascular morphogenesis. Methods Mol Biol 1214: 49-66, 2015.
13. Horsman MR and Vaupel P: Pathophysiological basis for the formation of the tumor microenvironment. Front Oncol 6: 66, 2016.

14. Patel A and Sant S: Hypoxic tumor microenvironment: Opportunities to develop targeted therapies. Biotechnol Adv 34: 803-812, 2016.

15. Sancakdar E, Guven AS, Uysal EB, Deveci K and Gültürk E: Important of angiopoietic system in evaluation of endothelial damage in children with crimean-congo hemorrhagic fever. Pediatr Infect Dis J 34: e200-e205, 2015.

16. Rogers TL and Holen I: Tumour macrophages as potential targets of bisphosphonates. J Transl Med 9: 177, 2011.

17. Sakurai T and Kudo M: Signaling pathways governing tumor angiogenesis. Oncology 81 (Suppl 1): S24-S29, 2011.

18. Morello S, Pinto A, Blandizzi C and Antonioli L: Myeloid cells in the tumor microenvironment: Role of adenosine. Oncoimmunology 5: e1108515, 2015.

19. Ostrand-Rosenberg S, Sinha P, Beury DW and Clements VK: Cross-talk between myeloid-derived suppressor cells (MDSC), macrophages, and dendritic cells enhances tumor-induced immune suppression. Semin Cancer Biol 22: 275-281, 2012.

20. Fukuda K, Kobayashi A and Watabe K: The role of tumor-associated macrophage in tumor progression. Front Biosci (Schol Ed) 4: 787-798, 2012.

21. Harris AL: Hypoxia-a key regulatory factor in tumour growth. Nat Rev Cancer 2: 38-47, 2002.

22. Ji RC: Hypoxia and lymphangiogenesis in tumor microenvironment and metastasis. Cancer Lett 346: 6-16, 2014.

23. Lee HJ and Jo DY: The role of the CXCR4/CXCL12 axis and its clinical implications in gastric cancer. Histol Histopathol 27: 1155-1161, 2012.

24. Henegan JC Jr and Gomez CR: Heritable cancer syndromes related to the hypoxia pathway. Front Oncol 6: 68, 2016.

25. Rapisarda A and Melillo G: Overcoming disappointing results with antiangiogenic therapy by targeting hypoxia. Nat Rev Clin Oncol 9: 378-390, 2012.

26. Alahuhta I, Aikio M, Väyrynen O, Nurmenniemi S, Suojanen J, Teppo S, Pihlajaniemi T, Heljasvaara R, Salo T and Nyberg P: Endostatin induces proliferation of oral carcinoma cells but its effect on invasion is modified by the tumor microenvironment. Exp Cell Res 336: 130-140, 2015.

27. Chen X, Zhang H, Zhu H, Yang X, Yang Y, Yang Y, Min H, Chen G, Liu J, Lu J, et al: Endostatin combined with radiotherapy suppresses vasculogenic mimicry formation through inhibition of epithelial-mesenchymal transition in esophageal cancer. Tumour Biol 37: 4679-4688, 2016.

28. Alevizakos M, Kaltsas S and Syrigos KN: The VEGF pathway in lung cancer. Cancer Chemother Pharmacol 72: 1169-1181, 2013.

29. Hato T, Zhu AX and Duda DG: Rationally combining anti-VEGF therapy with checkpoint inhibitors in hepatocellular carcinoma. Immunotherapy 8: 299-313, 2016. 